\title{
Reingreso por infección de sitio quirúrgico: una revisión integradora
}

\author{
Lilian Machado, Ruth N. T. Turrini y Ana L. Siqueira
}

\author{
Universidade FUMEC, Belo \\ Horizonte, MG, Brasil. \\ Facultad de Ciencias Humanas, \\ Sociales y Salud (LM) \\ Escola de Enfermagem da \\ Universidad de São Paulo \\ Brasil. \\ Departamento de Enfermería \\ Médico-Quirúrgica (RNTT, ALS). \\ Conflictos de interés: no hay. \\ Financiamiento: no hubo. \\ Recibido: 27 de marzo de 2012 \\ Aceptado: 8 de diciembre de 2012 \\ Correspondencia a: \\ Lilian Machado Torres \\ lilian.torres@superig.com.br
}

\section{Introducción}

$\mathrm{L}$ a infección del sitio quirúrgico (ISQ) es una de las causas más frecuentes de complicaciones en el período post-operatorio. Se considera un riesgo inherente a la realización de cualquier acto quirúrgico y está asociada a un conjunto de factores relacionados con el propio procedimiento, así como a las condiciones físicas y clínicas del paciente ${ }^{1}$.

Las ISQ son las infecciones de ocurrencia más frecuente en pacientes quirúrgicos, alcanzando a $38 \%$ de todas las infecciones asociadas a la atención de salud (IAAS) en un estudio conducido por los Centers for Disease Control and Prevention (CDC) $)^{2}$, entre 1986 y 1996. En una investigación reciente realizada en Inglaterra, Gales e Irlanda del Norte, de febrero a mayo de 2006, en pacientes adultos operados, se identificó que la ISQ fue la tercera causa más importante entre las infecciones ocurridas, con $14,5 \%$ de los casos ${ }^{3}$.

Un estudio brasileño que estimó la magnitud de las IAAS en hospitales de nivel terciario, realizado en 1994 por la Coordinación de Control de Infección Hospitalaria del Ministerio de Salud, incluyó 8.624 pacientes en 99 hospitales y señaló a la ISQ como la segunda infección de mayor prevalencia $(15,6 \%)$ después de la neumonía $(28,9 \%)^{4}$. La tasa promedio de ISQ en herida limpia entre los hospitales que participan del Sistema de Vigilancia de las Infecciones Hospitalarias del Estado de Sao Paulo fue de $0,94 \%$ (DP: 1,47 ) con una variación de 0,29 a 3,12\%, dependiendo de la especialidad quirúrgica ${ }^{5}$.
El riesgo para adquirir una infección aumenta según la gravedad del paciente y la complejidad del cuidado; en los pacientes quirúrgicos, este riesgo se intensifica debido a la potencial contaminación inherente a todo procedimiento invasor.

El tiempo de internación para la realización de la cirugía es relativamente corto $\mathrm{y}$, generalmente, el individuo recibe el alta en las siguientes $72 \mathrm{~h}$ después del acto operatorio. Dada esta particularidad, el paciente no permanece internado el tiempo suficiente para el diagnóstico de una ISQ, el que sólo será posible consignar si el hospital posee un sistema de vigilancia de infecciones post-alta, si el individuo fuera reingresado por una complicación quirúrgica o si hubiera una notificación del evento por parte del médico o enfermero durante la consulta ambulatoria de control post-alta.

La vigilancia epidemiológica post-alta de las ISQ, definida como la observación activa, sistemática y continua de los pacientes, aunque se recomienda en documentos oficiales ${ }^{6}$, ofrece una serie de dificultades para su ejecución. Éstas se relacionan con la falta de recursos humanos para mantener ese sistema, problemas en contactar al paciente después del alta por cambio de domicilio o número de teléfono, consulta de control postalta del paciente en clínicas particulares u otros centros de salud que no notifican la ISQ a la institución de origen.

Un estudio sobre vigilancia de las IHO en niños y adolescentes en un hospital universitario de Belo Horizonte (Minas Gerais, Brasil) $^{7}$ demostró que la inclusión de las infecciones relacionadas con el procedimiento quirúrgico 
ocurridas después del alta aumentaron la tasa de ISQ desde 7,5 a $11,9 \%$, lo que sugiere que la ocurrencia de infección podría ser subestimada en muchos estudios de prevalencia.

El esfuerzo para mantener la vigilancia de ISQ en el período post-alta se ha ampliado $^{7}$; sin embargo, dada sus dificultades, ya sea por factores de infraestructura y recursos humanos institucionales, o por factores relacionados con el retorno de información por parte de los pacientes, los reingresos hospitalarios pueden ser una fuente importante de información, al menos, en relación a las ISQ de mayor gravedad.

Entendemos el reingreso como una internación posterior en la misma institución hospitalaria, después del egreso. Sin embargo, no existe un consenso en la literatura médica sobre el plazo post-alta para considerar una admisión como reingreso. En el caso de las ISQ, ese rango está asociado a la propia definición de ISQ postalta, que incluye las infecciones ocurridas hasta 30 días después del egreso o hasta un año en el caso de implantes o prótesis, cuando están relacionadas con el procedimiento quirúrgico ${ }^{2}$.

Los datos de reingreso por ISQ podrían ser una fuente de información e indicadores de calidad de los resultados del acto quirúrgico, principalmente, en aquellos procedimientos que tienen mayor riesgo para desarrollar una infección ${ }^{8}$. Considerando la creciente preocupación de los profesionales en la prevención de las IAAS, este estudio analizó la literatura científica sobre los reingresos hospitalarios por ISQ.

\section{Material y Métodos}

Se efectuó una revisión integradora de la literatura médica sobre el reingreso por ISQ. La revisión integradora consiste en un método generador de conocimientos actuales sobre el problema en estudio, que pueden ser validados y transferidos a la práctica ${ }^{9}$.

Para la elaboración de esta revisión se siguieron las siguientes etapas: identificación del tema y de la pregunta de la investigación, establecimiento de criterios de inclusión, de exclusión y búsqueda en la literatura científica, definición de las informaciones necesarias en cada estudio, evaluación de los estudios incluidos en la revisión integradora, interpretación de los resultados y síntesis de los conocimientos9.

La pregunta formulada para la investigación fue: ¿Cuáles son las características y la frecuencia de los reingresos por ISQ? Fueron incluidos todas las publicaciones que abordaron el reingreso por ISQ, en los idiomas portugués, inglés, español, francés e italiano; y excluidos estudios en niños y publicaciones no disponibles en la versión electrónica o en las bibliotecas brasileñas.

La investigación fue realizada en la base de datos ME-
DLINE, LILACS, SCOPUS y Cochrane Library con las siguientes palabras clave: patient readmission (reingreso del paciente); surgical wound infection (infección de herida operatoria) y cross infection (infección hospitalaria). Como la base de datos más antigua, MEDLINE, que incorpora artículos desde 1966, se consideró el período de búsqueda desde el año 1966 a 2010.

En primer lugar, los estudios fueron analizados por el título y resumen y excluidos aquellos que no reunían los criterios de inclusión. Posteriormente, los textos seleccionados fueron leídos en su totalidad.

Los artículos fueron analizados en relación al tipo de estudio, año de publicación, objetivos, muestra y aspectos epidemiológicos de las ISQ responsables por el reingreso de pacientes, como tipo de cirugía, tiempo de internación, tiempo para reingreso, complicaciones quirúrgicas y agente etiológico.

\section{Resultados}

En las bases de datos se encontraron 206 artículos, siendo 105 de la base de datos MEDLINE, 100 del SCOPUS, uno del LILACS y ninguno en Cochrane Library. Después de la lectura de los resúmenes, fueron seleccionados 48 artículos para lectura completa, de los cuales 13 artículos cumplían los criterios de inclusión del estudio.

En los trabajos seleccionados, se constató mayor número de publicaciones en el continente americano, siendo seis de los E.U.A ${ }^{10-15}$ y dos de Canadá ${ }^{16-17}$, de Europa hubo cinco publicaciones ${ }^{8,18-21}$ y en Oceanía, una $^{22}$. En relación al período de publicación, desde 1993 a 2009 , ocho $(57,1 \%)$ fueron publicados en la década de 2000 (Tabla 1).

Hubo predominio de estudios transversales ${ }^{8,13,15,16,19,21,22}$ : cuatro de cohortes ${ }^{10-12,17}$, en dos de ellos los pacientes fueron pareados según edad, índice de riesgo quirúrgico, fecha de cirugía, tipo de procedimiento y cirujano ${ }^{10,11} \mathrm{y}$ dos fueron longitudinales ${ }^{14,18}$.

Tres estudios fueron multicéntricos ${ }^{8,11,20} \mathrm{y}$ el número de instituciones involucradas varió de dos a nueve. Uno de los estudios utilizó la base de datos Health PersonOriented Information Database, mantenido por el gobierno de Canadá ${ }^{16}$. La duración media de los estudios fue de 61,8 meses (variación de 12 a 156 meses).

Cuatro estudios $(28,6 \%)$ involucraron procedimientos ortopédicos ${ }^{14,18,21,22}$; cuatro $(28,6 \%)$ fueron de intervenciones quirúrgicas sin especificar la especialidad $d^{8,10,11,19}$; dos $(14,3 \%)$ fueron de cirugía cardíaca ${ }^{13,17}$; uno $(7,1 \%)$ de intervenciones vasculares ${ }^{20}$; uno $(7,1 \%)$ de mastectomías o reconstrucciones mamarias ${ }^{12}$; uno $(7,1 \%)$ de lifting facial ${ }^{15}$ y uno $(7,1 \%)$ de colecistectomías, apendicectomías e histerectomías ${ }^{16}$.

La ISQ fue objeto de investigación en nueve publicaciones $^{10-13,15-17,18,20}(64,3 \%)$, tres de ellos evaluaron también 
Tabla 1. Estudios seleccionados según autores, año de publicación, país, tipo de estudio, muestra y período

\begin{tabular}{|c|c|c|c|c|c|}
\hline Autores & Año & Tipo & País & Muestra & Período \\
\hline Fiorani et $\mathrm{al}^{20}$ & 1993 & Transversal & Italia & $\begin{array}{l}\text { Un hospital: } 37 \text { pacientes reingresados post-injertos vasculares de Dacron para aneurisma } \\
\text { de aorta o enfermedad aorto-iliaca oclusiva }\end{array}$ & oct/1987 a nov/1991 \\
\hline LeRoy et al $^{15}$ & 1994 & Transversal & E.U.A. & Un hospital: 11 pacientes reingresados com ISQ post lifting facial & 1984 a 1991 \\
\hline Goulet et al ${ }^{14}$ & 1997 & Longitudinal & E.U.A. & $\begin{array}{l}\text { Un hospital: } 170 \text { pacientes } \geq 18 \text { años con uno o más injertos de hueso de cresta ilíaca } \\
\text { (3 reingresos) }\end{array}$ & 1988 a 1991 \\
\hline Bedi et $a^{22}$ & 1997 & Transversal & Australia & Un hospital: 33 reingresados hasta 12 meses post-artroplastía de rodilla & jul/1989 a dic/1994 \\
\hline Borger et $\mathrm{al}^{17}$ & 1998 & Cohorte & Canadá & $\begin{array}{l}\text { Un hospital: } 309 \text { pacientes con ISQ post-esternotomía, } 92 \text { necesitaron de una reoperación } \\
\text { (23 reingresos) }\end{array}$ & ene/1990 a dic/1995 \\
\hline Kirkland et al ${ }^{10}$ & 1999 & $\begin{array}{l}\text { Cohorte } \\
\text { pareado }\end{array}$ & E.U.A. & $\begin{array}{l}\text { Un hospital: } 255 \text { pares - pareado por edad, procedimiento, fecha de la cirugía, puntuación } \\
\text { de riesgo quirúrgico y cirujano ( } 94 \text { reingresaron por } \mathrm{IHO})\end{array}$ & jun/1991 a jul/1995 \\
\hline Olsen et $\mathrm{al}^{13}$ & 2003 & Transversal & E.U.A. & $\begin{array}{l}\text { Un hospital-banco de datos STS Adult Cardiac Surgery Database: } 1980 \text { pacientes de } \\
\text { revascularización miocárdica; } 75 \text { ISQ en safenectomía (39 reingresos) }\end{array}$ & ene/1996 a jun/1999 \\
\hline Rotermann $^{16}$ & 2004 & Transversal & Canadá & $\begin{array}{l}\text { Banco de datos: } 8.323 \text { pacientes con ISQ post-colecistectomía, histerectomía e apendice- } \\
\text { tomía (3.554 reingresados por infecciones) }\end{array}$ & abr/1997 a mar/2000 \\
\hline Engbaek et $\left.a\right|^{8}$ & 2006 & Transversal & Dinamarca & $\begin{array}{l}\text { Dos hospitales: } 18.736 \text { procedimientos cirugía de día; } 113 \text { pacientes reingresados por } \\
\text { complicaciones quirúrgicas }\end{array}$ & 5 años \\
\hline Cullen et $\mathrm{al}^{21}$ & 2006 & Transversal & Reino Unido & Un hospital: 60 reingresos post-artroplastía total de cadera & ago/1997 a mar/2001 \\
\hline Olsen et $\mathrm{al}^{12}$ & 2008 & Cohorte & E.U.A & $\begin{array}{l}\text { Un hospital: } 949 \text { pacientes sometidos a mastectomía o cirugía de reconstrucción de } \\
\text { mamas; } 50 \text { ISQ ( } 49 \text { reingresos) }\end{array}$ & jul/1999 a jun/2002 \\
\hline $\begin{array}{l}\text { Marang-van de } \\
\text { Mheen et al }{ }^{19}\end{array}$ & 2008 & Transversal & Países Bajos & $\begin{array}{l}\text { Un hospital: } 1.960 \text { pacientes quirúrgicos: } 487 \text { (24,8\%) evento adverso; } 147 \text { (7,5\%) re- } \\
\text { ingresos }\end{array}$ & 2003 \\
\hline Kaye $^{11}$ & 2009 & $\begin{array}{l}\text { Cohorte } \\
\text { pareado }\end{array}$ & E.U.A & $\begin{array}{l}\text { Ocho hospitales: pacientes } \geq 65 \text { años }-561 \text { casos de ISQ (68,3\% reingresados) y } 576 \\
\text { controles sin ISQ ( } 13,5 \% \text { reingresados) }\end{array}$ & 1991 а 2003 \\
\hline
\end{tabular}

el costo ${ }^{14-16}, \mathrm{y}$ las complicaciones postoperatorias en cinco $(35,7 \%)^{8,14,19,21,22}$.

Parte de los estudios tuvo como objetivo principal analizar el reingreso ${ }^{15,20,21-22}$. El primero incluyó 11 pacientes readmitidos por ISQ post-lifting de rostro ${ }^{15}$; el segundo se propuso verificar la eficacia de la cintigrafía con tecnecio para diagnosticar la ISQ en los injertos vasculares de Dacron ${ }^{\circledR}$ en 37 pacientes post-quirúrgicos de aneuris$\mathrm{ma}^{20}$; el tercero investigó las razones para el reingreso de emergencia de 60 pacientes hasta 28 días después de la artroplastía total de cadera $^{21} \mathrm{y}$, el último analizó los reingresos de 33 pacientes hasta un año después de la artroplastía primaria de rodilla ${ }^{22}$.

El tiempo transcurrido entre el procedimiento y el aparición de la ISQ varió en los diferentes estudios, con una mediana de 11 a 25 días ${ }^{12,17,18}$ y media de 6,3 meses en procedimientos cardiovasculares con implante de injerto de Dacron ${ }^{\circledR}$ para aneurismas abdominales ${ }^{20}$. Sin embargo, algunos estudios delimitaron el tiempo de observación; en el estudio de eventos adversos se realizó una entrevista a los pacientes cuatro semanas después del alta ${ }^{19} \mathrm{y}$ el estudio del impacto de la ISQ en la mortalidad, hospitalización y costos siguió a los pacientes hasta 90 días después de la cirugía ${ }^{11}$. Los demás estudios hicieron la observación conforme el tiempo limitado para el estudio.

El tiempo mencionado para definir el reingreso fue diferente entre los estudios: 28, 30, 60 y hasta 90 días después de la cirugía ${ }^{8,16,21,22}$, lo que compromete las comparaciones de las tasas de IHO.

El tiempo de internación hospitalaria, incluida la primera admisión hospitalaria y las consiguientes por complicaciones quirúrgicas, varió de una media de 5,5 a 32 días en los estudios analizados que presentaron esta información ${ }^{10,11,16,17}$.

La tasa de reingreso por ISQ en los trabajos sobre eventos adversos en el post-operatorio fue de 2,5 a $3 \%$, con excepción del estudio de artroplastía de rodilla ${ }^{22}$ (Tabla 2).

En el estudio de artroplastía de rodilla ${ }^{22}$, aunque hayan sido consideradas 14 complicaciones infecciosas, nueve $(64,3 \%)$ fueron tratadas empíricamente con antimicrobianos por la ausencia de patógenos aislados en los cultivos microbiológicos realizados.

Las tasas de reingreso por ISQ estuvieron debajo de 5\% en la mayoría de los estudios (Tabla 3). Las mayores tasas fueron observadas en el estudio sobre ISQ en miembros inferiores, en los pacientes sometidos a revascularización de miocardio ${ }^{13}$ y en aquellos de cirugías de mama ${ }^{12}$; en este último, incluyeron algunos pacientes con cáncer de mama.

En los estudios pareados no fue posible calcular la tasa de reingreso, pero la proporción de reingreso en los pacientes con ISQ (40 y 68,3\%) fue mayor que en el grupo $\sin$ ISQ $(7,4 \text { y } 13,5 \%)^{10,11}$.

La presencia de ISQ fue responsable de otras intervenciones como cirugías adicionales ${ }^{15,19}$, incluyendo remo- 
Tabla 2. Síntesis de los datos de reingreso e ISQ de los estudios sobre complicaciones o eventos adversos

\begin{tabular}{|c|c|c|c|c|c|}
\hline Tipo de procedimiento & $\begin{array}{c}\text { Paciente } \\
\text { n }\end{array}$ & $\begin{array}{l}\text { Paciente con } \\
\text { complicación } \\
\mathrm{n} \quad(\%)\end{array}$ & $\begin{array}{l}\text { Paciente con complicación } \\
\text { reingresado } \\
\mathbf{n}(\%)\end{array}$ & $\begin{array}{c}\text { Paciente reingreso por } \\
\text { ISQ } \\
n \quad \text { (\%) }\end{array}$ & $\begin{array}{l}\text { Tasa de reingresos por } \\
\text { ISQ } \\
\text { (\%) }\end{array}$ \\
\hline Artroplastía de cadera ${ }^{21}$ & $\ldots$ & $\ldots$ & 768 & $(0,1)$ & $\ldots$ \\
\hline Injerto de hueso de cresta ilíaca ${ }^{14}$ & 170 & $41 \quad(24,1)$ & $(1,0)$ & $4(100)$ & 2,4 \\
\hline Cirugías de día ${ }^{8}$ & 16.048 & $113(7,0)$ & $113(100)$ & $41 \quad(36,3)$ & 2,6 \\
\hline No especificado ${ }^{19}$ & 1.960 & $487(24,8)$ & $147 \quad(30,2)$ & $64 \quad(43,5)$ & 3,3 \\
\hline Artroplastía de rodilla22 & 160 & $\ldots$ & $33(\ldots)$ & $14 \quad(42,4)$ & 8,8 \\
\hline
\end{tabular}

Tabla 3. Síntesis de los datos de ISQ y reingreso en los estudios sobre ISQ

\begin{tabular}{|c|c|c|c|c|}
\hline Tipo de procedimiento & $\begin{array}{l}\text { Paciente } \\
\text { n }\end{array}$ & $\begin{array}{l}\text { Paciente con ISQ } \\
\text { n (\%) }\end{array}$ & $\begin{array}{l}\text { Paciente con ISQ reingresado } \\
\qquad \begin{array}{l}\mathrm{n}(\%)\end{array}\end{array}$ & $\begin{array}{c}\text { Tasa de reingresos por ISQ } \\
(\%)\end{array}$ \\
\hline Injerto aórtico de Dacron²0 & $\ldots$ & 37 & $37(100)$ & $\ldots$ \\
\hline Lifting facial ${ }^{15}$ & 6.166 & $\ldots$ & $11(\ldots)$ & 0,2 \\
\hline Revascularización miocárdica ${ }^{17}$ & 12.267 & $92(0,7)$ & $21 \quad(22,8)$ & 0,2 \\
\hline Colecistectomía, apendicectomía, histerectomía ${ }^{16}$ & 382.287 & $8.323(2,2)$ & $3.554 \quad(42,7)$ & 0,9 \\
\hline Revascularización miocárdicaa ${ }^{13^{*}}$ & 1980 & $75(3,8)$ & $39 \quad(52)$ & 2 \\
\hline $\begin{array}{l}\text { Artroplastía de rodilla } \\
\text { Artroplastía de cadera } \\
\text { Síntesis de fractura de fémur }{ }^{18}\end{array}$ & $\begin{array}{l}3.706 \\
6.207 \\
1.899\end{array}$ & $\begin{array}{rr}84 & (2,3) \\
244 & (3,9) \\
56 & (2,9)\end{array}$ & $\begin{array}{rr}14 & (16,7) \\
133 & (54,5) \\
21 & (37,5)\end{array}$ & $\begin{array}{l}0,4 \\
2,1 \\
1,1\end{array}$ \\
\hline Cirugía de mama ${ }^{12}$ & 949 & $50 \quad(5,3)$ & $48 \quad(96)$ & 5,1 \\
\hline
\end{tabular}

ción de implantes ${ }^{12}$, desbridamiento ${ }^{12,14}$, re-intervenciones abiertas post-cirugías cerradas ${ }^{8}$, reconstrucción de pared con injerto ${ }^{17}$, manipulación o biopsias tardías ${ }^{22}$.

Los estudios que presentaron análisis microbiológico de las ISQ evidenciaron el predominio del género Staphylococcus y, entre ellos, Staphylococcus coagulasa negativa $^{15,17,18,21}$ y $S$. aureus ${ }^{10-12,13,15-17}$. Otras cocáceas grampositivas y bacilos gramnegativos también fueron identificados ${ }^{10,13,15}$. En el estudio de ISQ en pacientes de la tercera edad, $58,2 \%$ de los aislados de $S$. aureus eran resistentes a meticilina ${ }^{11}$.

\section{Discusión}

La incidencia habitual de las ISQ probablemente no refleja la realidad, dada la corta permanencia institucional del paciente, lo que restringe la vigilancia epidemiológica de las ISQ para el período de hospitalización ${ }^{7}$, y hace frecuente el diagnóstico de ISQ en el reingreso ${ }^{10,12-17,19,21-22}$. La vigilancia post-alta podría detectar hasta $84 \%$ de los diagnósticos de las ISQ ${ }^{2}$, principalmente en las cirugías de corta estadía y representa el punto central de un programa efectivo de control de IAAS.

Los resultados de la vigilancia post-alta mejoran las tasas subestimadas y podrían ser punto de partida para la identificación de procedimientos que representan mayor riesgo para las ISQ, orientando las acciones de prevención y control ${ }^{23}$.

Un estudio de cohorte con pacientes sometidos a cirugías electivas, en la región de Ontario, Canadá, observó que $58 \%$ de las infecciones fueron diagnosticadas con posterioridad al alta y de ellas, $10,8 \%$ en el momento del reingreso y $23,1 \%$ a través del servicio de emergencia ${ }^{24}$.

Varios estudios han puesto de manifiesto la importancia de la vigilancia de las IAAS, no sólo por los datos, sino también para implementar acciones de prevención ${ }^{3,7}$, reducir costos asociados y morbilidad $^{25}$, y evitar los reingresos por ISQ. La literatura científica otorga un aumento promedio de 3 a 20 días de hospitalización total para los pacientes que desarrollan $\mathrm{ISQ}^{25}$, y las de mayor gravedad, con compromiso de músculos y fascia, tienen como consecuencia mayor frecuencia de reingresos ${ }^{18}$.

Con frecuencia las ISQ superficiales aparecidas apenas después del alta, no son notificadas. Aquellas infecciones más profundas, de órganos y cavidades tienen una detección más fiable ya que requieren evaluación profesional y reingreso, permitiendo una mayor proporción de notificaciones $^{26}$.

Los pacientes que se infectan, aumentan significativamente su estadía hospitalaria en la internación inicial ${ }^{10,13}$, y hospitalizaciones prolongadas aumentan el riesgo de reingreso, independientemente de la ocurrencia de eventos adversos intrahospitalarios, como fue observado en los estudios $^{11,16,19}$. 
El tiempo de aparición de la ISQ observado en los estudios es coherente con los tiempos descritos tanto en la literatura científica como en la propia definición de las ISQ ${ }^{2}$.

En ausencia de implantes, el tiempo promedio para el inicio de los signos y síntomas ha sido de alrededor de 15 días. Un estudio que analizó métodos de vigilancia post-operatoria en dos hospitales de Sao Paulo (Brasil) encontró $80 \%$ de las ISQ diagnosticadas hasta el $14^{\circ}$ día después del procedimiento ${ }^{27}$. En un hospital universitario italiano con tasa global de ISQ de 10,6\%, el diagnóstico de estas infecciones ocurrió 11,5 días después del procedimiento $^{23}$.

En ortopedia, específicamente en las artroplastías de rodilla y cadera, el tiempo de aparición de los signos y síntomas caracteriza la infección como temprana o tardía. Castella y cols. ${ }^{28}$, encontraron que $95,3 \%$ de las ISQ en prótesis de rodilla y cadera fueron diagnosticadas en los primeros 90 días, enfatizando la importancia de la vigilancia en los primeros tres meses como una buena estimación de una ISQ en implantes quirúrgicos.

Escasos estudios tuvieron como foco principal los reingresos por ISQ y las informaciones encontradas, de modo general, no siempre permitieron calcular la tasa de reingreso por ISQ. Las tasas encontradas en la mayoría de los estudios estuvieron debajo de 3,3\%, con excepción de uno de los estudios de artroplastía de rodilla ${ }^{22}$, y el de cirugías de mama ${ }^{12}$. En los estudios con pacientes sometidos a artroplastía de rodilla ${ }^{18,22}$, no se encontró algún factor que justificara la diferencia en las tasas observadas. El intervalo de observación post-quirúrgico podría explicar ese hallazgo, pero ambos estudios consideraron reingresos hasta un año después de la intervención quirúrgica.

Una de las dificultades en el estudio de los reingresos es definir el rango de tiempo que caracteriza un reingreso, aunque se considere que cualquier admisión después del alta por motivos relacionados a la primera hospitalización, independiente del tiempo, es un reingreso ${ }^{29}$. La definición de ISQ del $\mathrm{CDC}^{2}$, es bastante clara en relación a esos rangos de tiempo; sin embargo, se observó que en el caso de implantes quirúrgicos, si el tiempo de observación no se extiende hasta un año después de la intervención, las diferencias encontradas en las tasas pueden carecer de importancia en las comparaciones entre las instituciones. En la presente revisión se encontraron períodos de observación retrospectivos o prospectivos con variación de 28 días $^{21}$ a 12 meses $^{12,18,22}$.

La proporción de pacientes con ISQ reingresados presentó variaciones de $16,7^{19}$ a $100 \%{ }^{20}$, dependiendo del procedimiento, sin posibilidad de sacar conclusiones. Del mismo modo, no se puede hacer comentario alguno acerca de los factores relacionados al reingreso por ISQ porque los estudios no compararon pacientes readmitidos por ISQ con el grupo sin reingreso.
En relación a las tasas de ISQ presentadas en los estudios, éstas tampoco permiten comparaciones, debido a que no todos ellos distinguieron si las ISQ se habían manifestado durante la hospitalización o post-alta, en el caso de los pacientes no reingresados. Además, se observó que cuando el objetivo fueron las complicaciones, las tasas de reingreso por ISQ fueron mayores que en los estudios de ISQ o de reingresos.

Los reingresos por complicaciones de la hospitalización previa en el servicio de emergencia son de aproximadamente $25 \%$ y más de $80 \%$ de esos eventos adversos son secundarios a procedimientos quirúrgicos ${ }^{30}$.

La proporción de ISQ en relación a las complicaciones post-operatorias difirió según el tipo de procedimiento realizado; la menor proporción de complicaciones fue observada en pacientes con cirugías ambulatorias. Estos hallazgos podrían ser atribuidos al estado de salud de los pacientes y a la baja complejidad de los procedimientos realizados en la condición de cirugía ambulatoria.

En dos estudios se presentó una comparación de las tasas de pacientes reingresados con ISQ en relación a la tasa de ISQ durante la hospitalización ${ }^{17}$, o a la tasa de ISQ post-alta ${ }^{21}$. En ambos casos, las tasas presentadas fueron similares, con excepción de las artroplastías de rodilla y las colecistectomías, en que la tasa de reingreso por ISQ fue aproximadamente $50 \%$ menor en relación a la tasa de ISQ en la hospitalización ${ }^{17}$, y post-alta ${ }^{21}$, respectivamente. Uno de los estudios identificó que 50\% de las ISQ en el período estudiado ocurrió después del alta del paciente ${ }^{18}$.

Algunos autores afirman que la vigilancia de los reingresos por ISQ sería adecuada para cirugías ortopédicas y vasculares donde la hospitalización es más prolongada ${ }^{31}$.

Se observó que, incluso en el caso de los reingresos, los agentes etiológicos no difieren del resto de las ISQ. La literatura científica ${ }^{32}$, muestra que aproximadamente dos tercios de las ISQ asociadas a implantes son causados por Staphylococcus spp. Estos agentes están presentes en individuos sanos y normalmente colonizan la piel y la nariz, aunque un estudio reciente demostró colonización de la región inguinal, recto, tracto urinario y vagina ${ }^{33}$. Boethelmann y cols. ${ }^{33}$ proponen, como forma de reducir las ISQ por esos agentes, la higienización de la piel en el preoperatorio del paciente con gluconato de clorhexidina $2 \%$ por su acción antiséptica residual sobre la piel y el tratamiento con mupirocina, en el caso de pacientes identificados como portadores de $S$. aureus resistente a meticilina (oxacilina).

La guías National Healthcare Safety Network $\mathrm{NHSN}^{34}$, recomiendan algunas prácticas de control de infecciones relacionadas con los factores de riesgo extrínsecos a los cuales los individuos están expuestos en la realización de procedimientos quirúrgicos, estableciendo barreras en la cadena epidemiológica de transmisión de patógenos. Son prácticas relacionadas con los momentos 
que implican una intervención quirúrgica: el antes, el durante y el después del procedimiento.

En la fase que antecede al acto quirúrgico, en las Guías de Prevención de ISQ del CDC se destaca el baño preoperatorio, la remoción de pelos y la preparación pre-quirúrgica de la piel. En el período denominado intraoperatorio se incluyen: cuidados referentes al equipo, tales como la antisepsia de las manos; relacionados con el procedimiento, como tiempo de duración, profilaxis antimicrobiana, técnica quirúrgica, hemostasia insuficiente, falla en la eliminación de espacio muerto, presencia de material extraño en la herida operatoria y trauma tisular; así como, cuidados del ambiente y equipos, como ventilación de la sala operatoria y esterilización inadecuada de instrumentales. Para la fase post-operatoria menciona las curaciones de la herida operatoria ${ }^{2}$.

La ISQ implica costos adicionales para el cuidado de la salud ${ }^{8}$. Costos directos asociados a la hospitalización quirúrgica en el primer año posterior al procedimiento se incrementan en comparación a los pacientes no infectados ${ }^{12}$, y pueden duplicarse ${ }^{11}$. Un estudio observó que el costo directo atribuible a ISQ fue de US\$3.089 ${ }^{10}$. De acuerdo a la Health Statistics Division de Otawa, Canadá ${ }^{16}$, se estima un valor de US\$ 5.000 por reingreso, pudiendo totalizar un aumento que varía de US\$ 5.400.000 a 6.300 .000 por cada año para un país.

Los costos individuales que desarrollaron ISQ después del alta, comparados a los que no desarrollaron, mostraron un aumento de 2,9 veces en los costos por paciente ${ }^{35}$. Los autores de ese estudio consideran costos directos a aquellos de fácil medición, tales como: prolongación de la hospitalización, reingresos, cirugías adicionales, terapia antimicrobiana, procedimientos de laboratorio y radiológicos, costos de materiales y equipos hospitalarios, así como los gastos de servicios prestados.

\section{Conclusiones}

Los estudios identificados en la literatura científica fueron escasos, principalmente aquellos que se centraron exclusivamente en el reingreso por una ISQ. Sin embargo, se nota un mayor número de informes de reingresos en individuos que se sometieron a cirugías ortopédicas, probablemente debido a la mayor utilización de prótesis. Los autores caracterizaron las ISQ desde el punto de vista de las complicaciones post-quirúrgicas, tratamiento y repercusiones sobre los costos y tiempo de hospitalización. Los estudios no se centraron en discutir la causa de las infecciones, la relación de las ISQ con el procedimiento o prácticas asistenciales.

Por los estudios que presentaron la comparación de tasas de pacientes readmitidos por ISQ y sin reingreso, se puede plantear la hipótesis de que los reingresos de pacientes con ISQ representarían un aumento de por lo menos $50 \%$ en las tasas de ISQ.

La frecuente indicación de las cirugías ambulatorias, así como, la creciente reducción de los períodos de hospitalización pre y post-operatorios son de gran importancia para el paciente. Sin embargo, aunque esto favorece la disminución de las infecciones, por otro lado, también dificulta la identificación de las ISQ cuando no hay un sistema efectivo de vigilancia post-alta de las ISQ. La vigilancia de la tasa de reingresos atribuidas a ISQ podría contribuir para dimensionar las ISQ que ocurren post-alta, ya que los datos mostraron que aproximadamente la mitad de las infecciones hospitalarias que se manifiestan después del alta del paciente generan reingresos, principalmente aquellas de cavidades y espacios profundos y las relacionadas con implantes.

Las cirugías plásticas y con implantes son cirugías consideradas limpias según su posible contaminación. Todo el equipo que atiende al paciente debe esforzarse en el cuidado del paciente quirúrgico ya que las ISQ en procedimientos limpios son prevenibles e incluyen técnicas de asepsia quirúrgica y la preparación de la piel del paciente en el pre-operatorio.

La presente revisión estuvo sujeta a algunas limitaciones debido a la diversidad de metodologías utilizadas en los estudios y al hecho de que pocos estudios estudiaron el reingreso como objetivo principal. Más aún, ninguno de los estudios investigó factores de riesgo relacionados con el reingreso de pacientes con ISQ.

\section{Resumen}

Introducción: Las infecciones de sitio quirúrgico (ISQ) representan un riesgo inherente a la realización de cualquier acto quirúrgico, asociado a factores propios del procedimiento, así como a condiciones del paciente. Objetivo. Analizar, en una revisión integradora, los estudios que abordaran aspectos epidemiológicos relacionados con el reingreso de pacientes por ISQ. Método: Se efectuó una revisión en las bases LILACS, SCOPUS, COCHRANE e MEDLINE con selección de artículos publicados desde 1966 hasta 2010. Resultados: Se analizaron 13 estudios, siendo siete estudios transversales, cuatro de cohortes y dos longitudinales. Escasos estudios se restringieron a analizar exclusivamente los reingresos relacionados a la ISQ. El tiempo para definir el reingreso varió de 28 a 90 días después de la cirugía y hubo un mayor número de estudios relacionados con procedimientos ortopédicos. La tasa de reingreso por ISQ en los estudios fue inferior a 5\%. El principal agente aislado en las ISQ fue Staphylococcus aureus y Staphylococcus coagulasa negativas. Conclusión: La vigilancia de los reingresos por ISQ podría contribuir a dimensionar la ocurrencia de ISQ post-alta, ya que aproximadamente la mitad de las ISQ post-alta originaron reingresos a centros hospitalarios. 


\section{Referencias bibliográficas}

1.- Culver D H, Horan T C, Gaynes R P, Martone W J, Jarvis W R, Emori T G, et al. Surgical wound infection rates by wound class, operative procedure, and patient risk index. National Nosocomial Infections Surveillance System. Am J Med 1991; 91 (3B): 152S-7S.

2.- Mangram A J, Horan T C, Pearson M L, Silver L C, Jarvis W R. Guidelines for prevention of surgical site infection, 1999. Centers for Disease Control and Prevention (CDC) Hospital Infection Control Practices Advisory Committee. Am J Infect Control 1999; 27: 97-132.

3.- Smyth E T, McIlvenny G, Enstone J E, Emmerson A M, Humphreys H, Fitzpatrick F, et al. Four country healthcare associated infection prevalence survey 2006: overview of the results. J Hosp Infec 2008; 69: 230-48.

4.- Prade S S, Oliveira S T, Rodrigues R, Nunes F A, Netto E M, Felix J Q. Estudo brasileiro da magnitude das infecções hospitalares em hospitais terciários. Rev Contr Infec Hosp 1995; 2: 11-24.

5.- Brandão de Assis D, Madalosso G, Ferreira S A, Yassuda Y Y. Divisão de Infecção Hospitalar. Centro de Vigilância Epidemiológica "Prof. Alexandre Vranjac". Coordenadoria de Controle de Doenças. Secretaria de Estado da Saúde. São Paulo, SP, Brasil. Boletim Epidemiológico Paulista. Análise dos dados do Sistema de Vigilância de Infecção Hospitalar do Estado de São Paulo-ano 2009. Bepa, agosto 2010; 7 (80). Consultado 10 de enero de 2011. Disponible en: http://www.cve.saude.sp.gov.br/agencia/ bepa80_ih.htm

6.- Ministério da Saúde (BR). Portaria número 2.616 de 12 de maio de 1998. Resolve expedir diretrizes e normas para a prevenção e o controle de infecções hospitalares. Diário Oficial da União, Brasília (DF), 13 de maio de 1998. Consultado 10 de mayo de 2010. Disponible en: http://www.anvisa.gov.br/legis/ portarias/2616_98.htm

7.- Oliveira A C, Ciosak S I. Infecção de sítio cirúrgico em um hospital universitário: vigilância pós-alta e fatores de risco. Rev Esc Enferm USP 2007; 41: 258-63.

8.- Engbaek J, Bartholdy J, Hjortso N C. Return hospital visits and morbidity within 60 days after day surgery: a retrospective study of 18,736 day surgical procedures. Acta Anaesthesiol Scand 2006; 50: 911-9.

9.- Pompeo D A, Rossi L A, Galvão C M. Revisão integrativa: etapa inicial do processo de validação de diagnóstico de enfermagem. Acta Paul Enferm 2009; 22: 434-8.

10.- Kirkland K B, Briggs J P, Trivette S L, Wilkinson W E, Sexton D J. The impact of surgical site infections in the 1990s: attributable mortality, excess length of hospitalization, and extra costs. Infect Control Hosp Epidemiol 1999; 20: 725-30.

11.- Kaye K S, Anderson D J, Sloane R, Chen L F, Choi Y, Link K, et al. The effect of surgical site infection on older operative patients. J Am Geriatr Soc 2009; 57: 46-54.

12.- Olsen M A, Chu-Ongsakul S, Brandt K E, Dietz J R, Mayfield J, Fraser V J. Hospitalassociated costs due to surgical site infection after breast surgery. Arch Surg 2008; 143: 53-60.

13.- Olsen M A, Sundt T M, Lawton J S, Damiano R J Jr, Hopkins-Broyles D, Lock-Buckley P, et al. Risk factors for leg harvest surgical site infections after coronary artery bypass graft surgery. J Thorac Cardiovasc Surg 2003; 126: 992-9.

14.- Goulet J A, Senunas L E, DeSilva G L, Greenfield M L. Autogenous iliac crest bone graft. Complications and functional assessment. Clin Orthop Relat Res 1997; 339: 76-81.

15.- LeRoy J L Jr, Rees T D, Nolan W B 3rd. Infections requiring hospital readmission following face lift surgery: incidence, treatment, and sequelae. Plast Reconstr Surg 1994; 93 : 533-6.

16.- Rotermann M. Infection after cholecystectomy, hysterectomy or appendectomy. Health Rep 2004; 15: 11-23.

17.- Borger M A, Rao V, Weisel R D, Ivanov J, Cohen G, Scully H E, et al. Deep sternal wound infection: risk factors and outcomes. Ann Thorac Surg 1998; 65: 1050-6.

18.- Huotari K, Lyytikainen O; Hospital Infection Surveillance Team. Impact of postdischarge surveillance on the rate of surgical site infection after orthopedic surgery. Infect Control Hosp Epidemiol 2006; 27: 1324-9.

19.- Marang-van de Mheen P J, van Duijn-Bakker N, Kievit J. Adverse outcomes after discharge: occurrence, treatment and determinants. Qual Saf Health Care 2008; 17: 47-52.

20.- Fiorani P, Speziale F, Rizzo L, De Santis F, Massimi GJ, Taurino M, et al. Detection of aortic graft infection with leukocytes labeled with technetium 99m-hexametazime. J Vasc Surg 1993; 17: 87-95.

21.- Cullen C, Johnson D S, Cook G. Re-admission rates within 28 days of total hip replacement. Ann R Coll Surg Engl 2006; 88: 475-8.

22.- Bedi H S, Fletcher S F, Rush J H, Choong P F. An audit of early hospital readmission after primary knee joint replacement. Aust N Z J Surg 1997; 67: 340-2.

23.- Prospero E, Cavicchi A, Bacelli S, Barbadoro P, Tantucci L, D'Errico M M. Surveillance for surgical site infection after hospital discharge: a surgical procedure-specific perspective. Infect
Control Hosp Epidemiol 2006; 27: 1313-7.

24.- Daneman N, Lu H, Redelmeier D A. Discharge after discharge: predicting surgical site infections after patients leave hospital. J Hosp Infect 2010; 75: 188-94.

25.- Dimick J B, Chen S L, Taheri P A, Henderson W G, Khuri S F, Campbell D A Jr. Hospital costs associated with surgical complications: a report from the private-sector National Surgical Quality Improvement Program. J Am Coll Surg 2004; 199: 531-7.

26.- Morton A, Mengersen K, Waterhouse M, Steiner S, Looke D. Sequential analysis of uncommon adverse outcomes. J Hosp Infect 2010; 76: 114-8.

27.- de Oliveira A C, Carvalho D V. Evaluation of underreported surgical infection evidenced by post-discharge surveillance. Rev Lat Am Enfermagem 2007; 15: 992-97.

28.- Castella A, Argentero P A, Farina E C, Charrier L, Del Prever E M, Zotti C M; Piemonte Nosocomial Infection Study Group. Incidence of surgical-site infections in orthopaedic surgery: a northern Italian experience. Epidemiol Infect 2011; 139: 777-82.

29.- Castro M S M, Carvalho M S, Travassos C. Factors associated with readmission to a general hospital in Brazil. Cad Saúde Pública 2005; 21 : 1186-200.

30.- Bíscaro Valera R, Turrini R N T. Fatores relacionados à readmissão de pacientes em serviço hospitalar de emergência. Cienc Enferm 2008; 14: 87-95.

31.- Reilly J, Allardice G, Bruce J, Hill R, McCoubrey J. Procedure-specific surgical site infection rates and postdischarge surveillance in Scotland. Infect Control Hosp Epidemiol 2006; 27: 1318-23.

32.- Darouiche R O. Treatment of infections associated with surgical implants. N Engl J Med 2004; 350: 1422-9.

33.- Boethelmann M, Frei R, Fenner L, Dangel M, Flockiger U, Widmer A F. Highly effective regimen for decolonization of methicillinresistant Staphyloccus aureus carriers. Infect Control Hosp Epidemiol 2008; 29: 510-6.

34.- Centers for Disease Control and Prevention. The National Healthcare Safety Network (NHSN) Manual. Patient safety component protocol. Division of Healthcare Quality Promotion National Center for Preparedness, Detection and Control of Infectious Diseases Atlanta, GA, USA. March, 2009. Consultado: 12 de marzo de 2011. Disponible en: http://www.cdc.gov/nhsn/ PDFs/pscManual/pscManual_current.pdf.

35.- Perencevich E N, Sands K E, Cosgrove S E, Guadagnoli E, Meara E, Platt R. Health and economic impact of surgical site infections diagnosed after hospital discharge. Emerg Infect Dis 2003; 9: 196-203. 